\title{
DETECTION AND CLASSIFICATION OF 2D AND 3D HYPER SPECTRAL IMAGE USING ENHANCED HARRIS CORNER DETECTOR
}

\author{
S. PAVITHRA * A. KARTHIKEYAN † AND P.M. ANU $\ddagger$
}

\begin{abstract}
Image classification and visualization is a challenging task in hyper spectral imaging system. To overcome this issue, here the proposed algorithm incorporates normalized correlation into active corner point of an image representation structure to perform hasty recognition by matching algorithm. Matching algorithms can be of two major categories, based on correlation and based on its features based on correlation and on its feature detection. Proposed algorithms often ignore issues related to scale and orientation and also those to be determined during the localization step. The task of localization involves finding the right region within the search image and passing this region to the verification process. A Harris corner detector is an advanced approach to detect and extract a huge number of corner points in the input image. We integrate all the extracted corner points into a possible task to locate candidate regions in input image. In terms of detection and classification the proposed method has got better result.
\end{abstract}

Key words: Harris corner detector, pyramid Correlation, Sub Pixel Mapping, Auto-correlation matrix.

AMS subject classifications. $68 \mathrm{U} 10$

1. Introduction. In 2D and 3D reconstruction from hyper spectral images has seldom been addressed in the literature. This is a challenging problem because 3D models reconstructed from different spectral bands demonstrate different properties [16]. Hyper spectral picture contains an abundance of information, however deciphering them requires a comprehension of precisely what properties of ground materials are attempting to estimate, and how they recognize with the estimations really made by the hyper spectral sensor. The instruments which are used in creation of Hyper spectral pictures called imaging spectrometers. The progression of these brain boggling sensors has incorporated the converging of two related however unmistakable advancements. Spectroscopy is the examination of light that is released by or reflected from assets and its assortment in vivacity with wavelength and spectroscopy and the remote imaging of earth and planetary surfaces. Detecting Corner points are considered important features for feature extraction and detection. Corner detection is a low-level image processing technique that is widely used in different computer vision applications.

Fifteen years prior just phantom remote detecting specialists had entry to hyper spectral images or programming tools to exploit such pictures. Over the previous decade hyper spectral picture investigation has developed into a standout among the most capable and quickest developing innovations in the field of remote detecting. The improvement of hyper spectral sensors and programming to break down the subsequent image information. Nowadays, developed build up area recognition in light of nearby invariant components has uncovered promising results. Be that as it may, it needs some format building pictures for preparing and in this way experiences a high figuring multifaceted nature and memory necessity. In their later, a more straightforward technique is utilized. Be that as it may, since it exclusively relies on upon nearby elements for acknowledgment, it can regularly be excessively feeble of a sign, making it impossible to dependably identify the developed districts in complex satellite picture. For this experiment of Harris corner evaluation we used a 3.2 eight core processor with 16 GB of memory with a NVIDIA GeForce GTX970 GPU [19].

\footnotetext{
*Assistant Professor Department of Computer Science and Engineering,Veltech Multi Tech Dr.Rangarajan Dr.Sakunthala Engineering College, Email: Pavicse06@gmail.com

${ }^{\dagger}$ Associate Professor Department of Electronics and Communication Engineering, Veltech Multi Tech Dr.Rangarajan Dr.Sakunthala Engineering College

${ }^{\ddagger}$ Assistant Professor Department of Information technology, Veltech Multi Tech Dr.Rangarajan Dr.Sakunthala Engineering College
} 
2. Materials and Methods. In this paper, we have introduce an unsupervised method toward all the while finding developed regions from various high-determination satellite pictures. The proposed strategy incorporates two noteworthy segments. In the first strategy a probability capacity based way to deal with concentrate hopeful developed locales, in which an enhanced Harris operation is proposed. In the second strategy spectrum grouping and chart cut-based unsupervised clustering calculation are adopted and territory recognition. Hyper otherworldly remote detecting innovation has progressed essentially in the previous two decades in these techniques. Based on the extensive tests, the proposed approach demonstrates the following main advantages over the previous works. First, it can simultaneously detect built-up regions from multiple images, and our experiment results show that the performance of built-up area detection can be further improved by exploring the cues drawn from multiple images. Second, the entire process is highly automatic and requires no human interaction. Taking after an underlying arbitrary assignment of delicate pixel extents to rigid sub pixel double classes, the calculation works in a progression of emphases, the calculation step contain three stages [3].

In Hyper Geometrical, Statistical, and Sparse Regression-Based method introduced by Jose M. Bioucas Hyper ghastly instruments secure electromagnetic vitality scattered inside of their ground quick field view in several otherworldly channels with high ghostly resolution [4]. A new sub-pixel mapping calculation in light of a Backpropagation neural network system with a perception model by Liangpei Zhang. The blended pixel is a typical issue in remote detecting order. Despite the fact that the structure of these pixels for various classes can be evaluated with a pixel un-blending demonstrate, the yield gives no sign of how such classes are circulated spatially inside of these pixels [5]. Joining pixel swapping and forming techniques to improve superdetermination mapping by Yuan-Fong Su, Giles M. Foody-Combining super-determination strategies can build the exactness with which the state of articles might be portrayed from symbolism. This is shown with two ways to deal with brushing the molding and pixel swapping techniques for super-determination mapping for parallel order applications.[6]super-determination land spread example expectation utilizing a Hop field neural system by A.J Tatema, H.G. Lewis-Landscape design speaks to a key variable in administration and comprehension of nature, an in addition driving numerous ecological models. Remote detecting can be utilized to give data on the spatial example of area spread elements, however investigation and arrangement of such symbolism experiences the issue of class blending inside of pixels

In like manner four methods for highlights can be utilized as a part of picture handling systems: low-level visual features, local features, local-global features and biologically inspired features [11-12]. Even though these features classification are always based on the assumption that all pixels in the image are pure pixels, which is unreasonable in remotely sensed images. In [13] Fisher suggested that there are four types of mixed pixels in remotely sensed images as follows in the upcoming section.

1. In convoluted sub pixel - the overall size of the 2D and 3D object is smaller than the size of the pixel.

2. Identify the Region Of Interest (ROI) - we identify the boundary value of an image along with the sizes of two or more land-cover classes on the ground value may be larger than the sizes of the pixel value, but parts of their boundaries lie in a sub- pixel.

3. Inter grade method - a pixel value assign a space for a transition from a cluster of one class to another class.

4. Sub-Pixels: the width of a land-cover is thinner and length of the land-cover class may be longer than a pixel. Many sub pixel mapping methods have been proposed to tackle the different types of mixed pixels [15].

The proposed strategies is explained briefly about pixel-based techniques. These strategies attempt to measure the likelihood of every pixel to have a place with the conceivable classes by utilizing measurement measures construct just in light of ghastly properties. The (MaxVar) Maximum Likelihood Classification method stays a standout among the most well known strategies for RSI characterization. The likelihood of every pixel to have a place with each of the characterized classes in MaxVer figure.

2.1. Sub Pixel Mapping. The key issues present in sub pixel mapping are the means by which to decide an ideal sub pixel dispersion of every class in a pixel. A coarser division picture is acquired by a phantom un-mixing method, as the information picture and every pixel is separated into sub pixels, where $\mathrm{S}$ speaks to the scale component [9-10]. The quantity of sub pixels for every area spread class is then dictated by the 


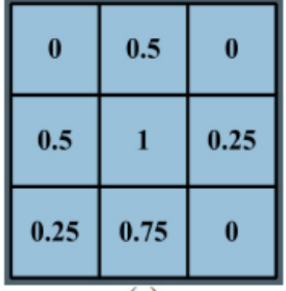

(a)

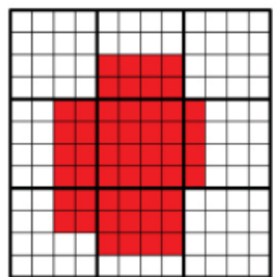

(b)

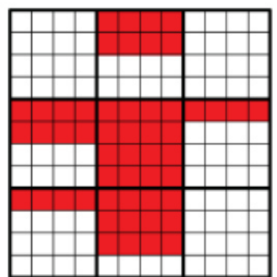

(c)

Fig. 2.1. To identify a $3 \times 3$ coarse pixels and possible distributions with (scale = 4, 2classes). (a) Detection of Fraction image. (b) Finding Optimal Distribution. (c) Inferior distribution

plenitudes of the portion picture. Fig. 2.1 delineates the rule of sub pixel mapping and depicts a basic case with two classes. In picture, as appeared in Fig. 2.1(a), assuming the scale division is represented as $\mathrm{S}$ and the value is 4 , a coarse pixel is isolated into $16(4 \times 4)$ sub pixels, and 0.5 in the portion picture implies that 8 $(16 \times 0.5)$ sub pixels fit in with area spread class 1. Fig. 2.1(b) and 2.1(c) depict two possible dispersion of sub pixels, and the previous is better than the last mentioned and uses that data to dole out the class with the most astounding likelihood. The upgrades in sensor advances have expanded the openness to high-determination pictures. Thus, new methodologies have been created to improve an utilization of the accessible information.

In the review of RSI characterization until 2005 is displayed in. Those works discuss about the difficulties and portrays every one of the strides that create the characterization process. Different arrangement strategies are introduced and gathered by scientific classification. All the more as of late another pattern can be watched. Numerous studies consider data encoded in locales [16]. In this strategy, districts were ordered by utilizing Mahalanobis separation and Support Vector Machines (SVM). In pattern recognition SVM is a class of algorithm made a locale based order strategy for high determination pictures that abused two approaches: In Lee et al., [15] MaxVer with area means and MaxVer with Gaussian Density Function were adopted and interpreted. Both mechanism shoes preferable results over pixel-based classifiers. Yu et al [16] likewise proposed a strategy to order RSI taking into account areas. The picture division and characterization were performed by utilizing fractal systems and non-parametric K-Nearest Neighbor individually. Another late work in this exploration territory was produced by Katartzis et al [17]. who proposed an area based RSI arrangement technique that uses Hierarchical Markov Models.

\section{Proposed Method.}

3.1. Harris Corner Detector. The main challenges present in the Harris Corners Detector (HCD) are used to identify corners points present in the input image [18]. The proposed technique is used to identify vertical and horizontal edges with help of Sobel detector. Those edge detectors are then used to reduce the effect of noise present in the image. The subsequent edges distinguished esteems are then all things considered to frame a vitality delineate contains valleys and tops. The pinnacles show the nearness of a corner point without loss of all-inclusive statement and also we have chosen different gray scale 2D and 3D image as a data set to generate the output. Let this image be given by $I$. we will assume a grayscale 2-dimensional image is used. Let this image be given by $I$. Consider taking an image patch over the area $(u, v)$ and shifting it by $(x, y)$. The weighted sum of squared differences (SSD) between these two patches, denoted $S$, is given by equation 3.1 .

$$
S(x, y)=\sum_{u} \sum_{v} w(u, v)(I(u+x+y)-I(u, v))
$$

$$
\begin{aligned}
& I(x)=G_{\sigma}^{x} * I \\
& I(y)=G_{\sigma}^{y} * I
\end{aligned}
$$


2. It produce theproducts of derivates at every pixel

$$
\begin{aligned}
& I_{x 2}=I_{x} I_{x} \\
& I_{y 2}=I_{y} I_{y} \\
& I_{x y}=I_{x} I_{y}
\end{aligned}
$$

3. It generates the sum of the products of derivatives at each pixel

$$
\begin{aligned}
& S_{x 2}=G_{\sigma 1} I_{x 2} \\
& S_{y 2}=G_{\sigma 1} I_{y 2} \\
& S_{x y}=G_{\sigma 1} I_{x y}
\end{aligned}
$$

4. Identify the matrix fuction at each pixel of $\mathrm{H}(\mathrm{x}, \mathrm{y})$

$$
H(x, y)=\left[\begin{array}{ll}
S_{x 2}(x, y) & S_{x y}(x, y) \\
S_{x y}(x, y) & S_{y 2}(x, y)
\end{array}\right]
$$

5. It respond the detector at each pixel threshold where

$$
R=\operatorname{DET}(H)-K(\operatorname{Trace}(H))_{2}
$$

6. Threshold on value of R, Here we adopted the faster Non-maximal Suppression to the gradient magnitude. In canny approach the edge direction is reduced to any one of the four directions.

To complete this task for a given point, Its gradient is compared with that point of its $3 \mathrm{x} 3$ neighborhood.

If CM (candidate magnitude>neighborhood)

Then

$$
\text { Else }
$$

The edge strength is maintained

Discarded

3.1.1. Corners as Interest Points. There are several application requiring to relating two or more pictures keeping in mind. The aim is to concentrate extract interested corner point from the image. For example, in case of two dynamic edges in a video gathering taken from a moving camera can be associated is conceivable to concentrate data with respect to the profundity of articles in the earth and the pace of the camera. The beast power strategy for contrasting each pixel in the two pictures is computationally restrictive for the dominant part of uses. Instinctively, one can picture relating two pictures by coordinating just areas in the picture that are somehow fascinating.

A Number Of Criteria To Identify A Corner Detector (Figure 3.1)

1. At first All "true corners" should be detected and then

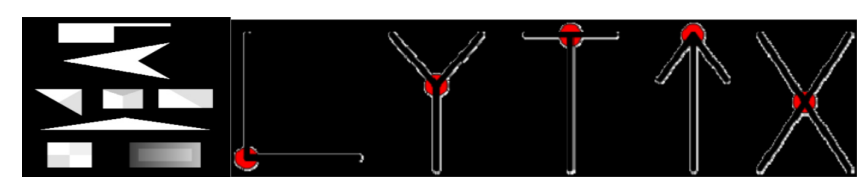

FIG. 3.1. Sample image with corner types of $L, Y, T$, Arrow, and X-junction 

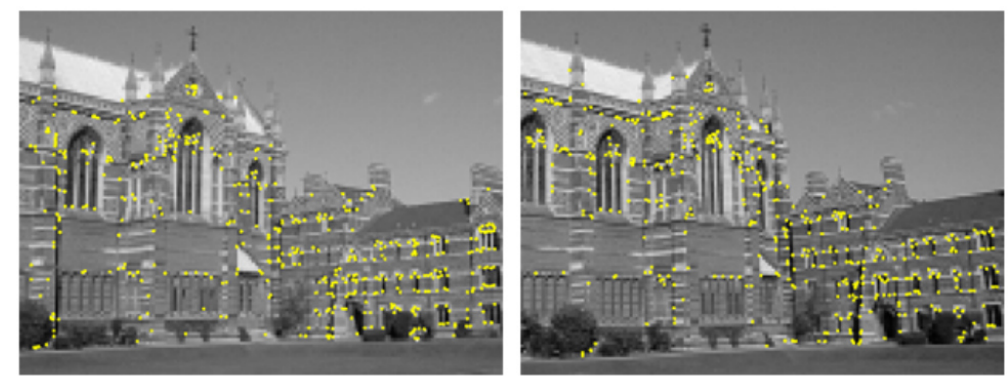

FIG. 3.2. Interest points extracted in $3 D$ building with Harris ( $\approx 500$ points)

2. No "false corners" should be detected.

3. All Corner points in the input image should be localized well.

4. Detector is supposed to have a high repeatability rate.

5. Detector is supposed to be robust

6. It is computationally more efficient.

Steps to extract corner points (Figure 3.2)

- It Extract the interest points with the proposed algorithm

- It compare the two points with cross-correlation

- Verification will be done with the fundamental matrix.

3.2. Determining Auto-correlation Matrix. It confine the structure of the local neighbourhood and gauge based on eigenvalues of $M$

$$
M=(\Delta x \Delta y)\left[\begin{array}{cc}
\sum_{\left(x_{k} y_{k}\right) \in W}\left(I_{x}\left(x_{k}, y_{k}\right)\right)^{2} & \sum_{\left(x_{k} y_{k}\right) \in W} I_{x}\left(x_{k}, y_{k}\right) I_{y}\left(x_{k}, y_{k}\right) \\
\sum_{\left(x_{k} y_{k}\right) \in W} I_{x}\left(x_{k}, y_{k}\right) I_{y}\left(x_{k}, y_{k}\right) & \sum_{\left(x_{k} y_{k}\right) \in W}\left(I_{y}\left(x_{k}, y_{k}\right)\right)^{2}
\end{array}\right]\left(\begin{array}{c}
\Delta x \\
\Delta y
\end{array}\right)
$$

The image has been calculated the interest point based on the eigenvalues of $\mathrm{M}$ with help of equation 3.12:

2 strong eigenvalues $=>$ interest point

1 strong eigenvalue $=>$ contour

0 eigenvalue $=>$ uniform region

The values of the pixels is compared with sum-square difference (SSD) of square neighbourhood about the points. This is the form of SSD measure:

$$
S S D=\sum \sum\left(W 1_{i, j}-W 2_{i, j}\right)^{2}
$$

4. Performance Evaluation. The experimental results and performance of the image classification are carried out based on the following result, initially the all the images done by preprocessing. The parameter that we use in the Harris algorithms was a Gaussian window with $W=3 \times 3$ kernel and $\sigma=0.3$ as the baseline algorithms. For the proposed technique, the pruning technique is first applied to the entire image in order to select the corner candidate set. Next, the corner measure of the corresponding baseline algorithm is applied to extract the final corners (Figures 4.1 and 4.2).

5. Conclusion. Detecting and classification of an 2D and 3D hyper spectral image, ccorner point extraction using enhance Harris corner detector and sub pixel mapping method has been adopted in this paper. The proposed technique concentrate on various hyper spectral pictures as dataset and otherworldly unmixing to meet with the genuine task; and also the ability to normalize and extract the interesting features with corner points in the input image by using enhanced Harris corner detector. By adding former information to obtain a better sub pixel mapping result. The conventional algorithms always utilize the fraction image obtained by the classification image sub sampling method as the input information. In Harris corner detector, the original image sub sampling is used to reduce the size of the image with original values and extract the interest point 

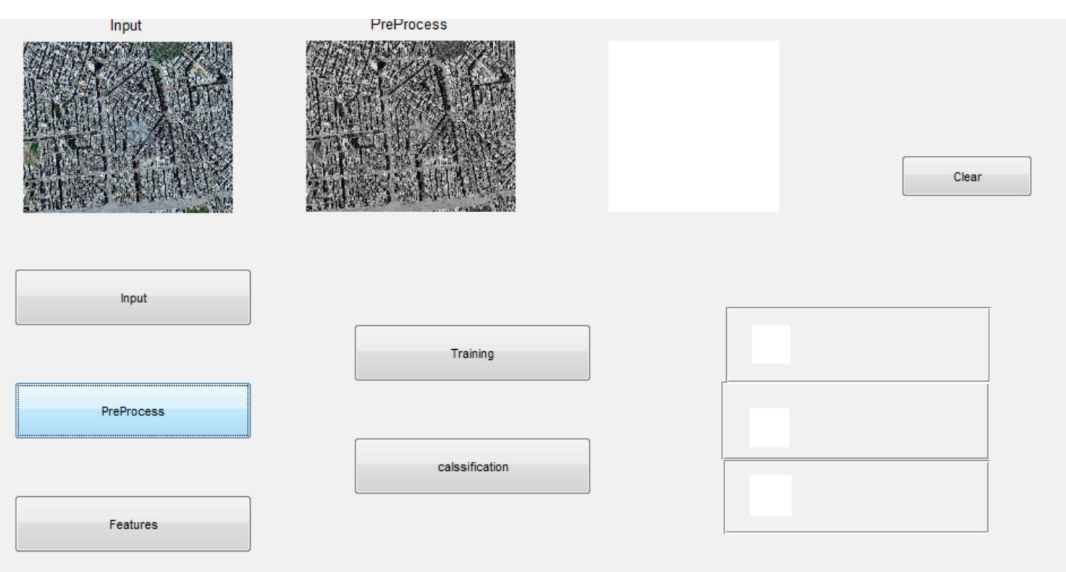

Features

(a)
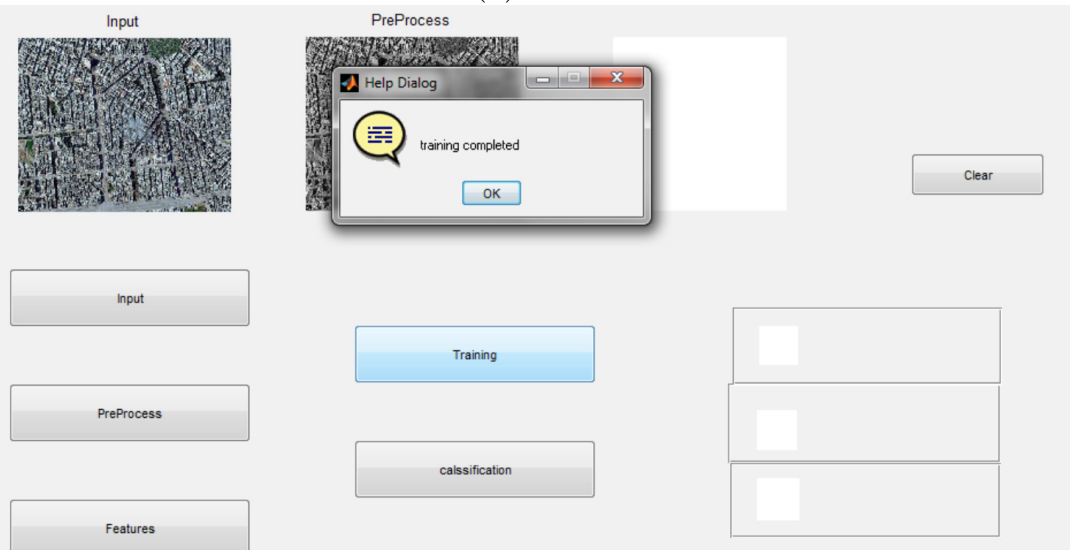

(b)
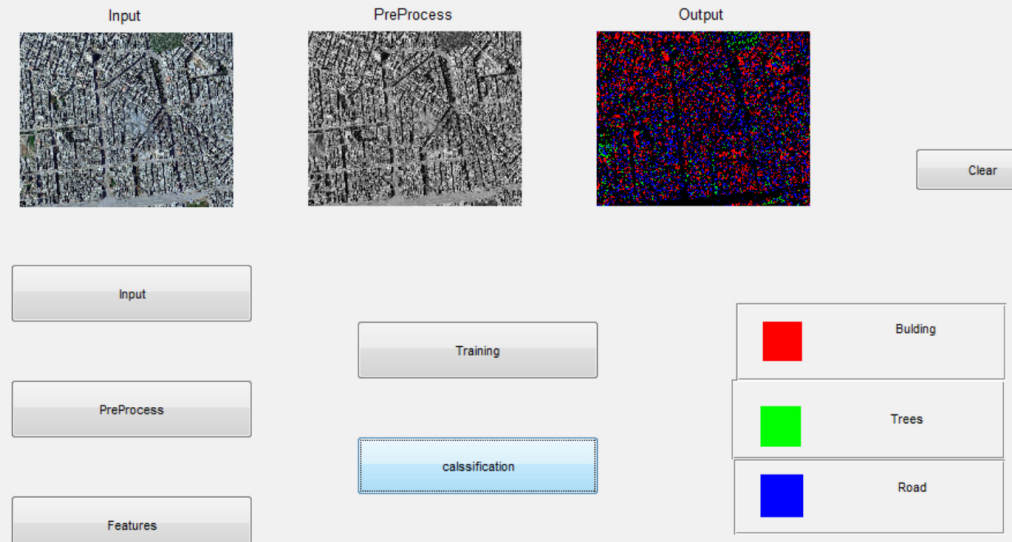

(c)

FIG. 4.1. (a) Initially input image loaded for training; (b) the input loaded and trained; (c) preprocessing done

as a result. The proposed method was create to be performs good accuracy but it requires more computational time. Therefore, in future it is suggested that the research should be carried out in the field of video mosaicing and other real time hyper spectral images using back propagation with neural network. 


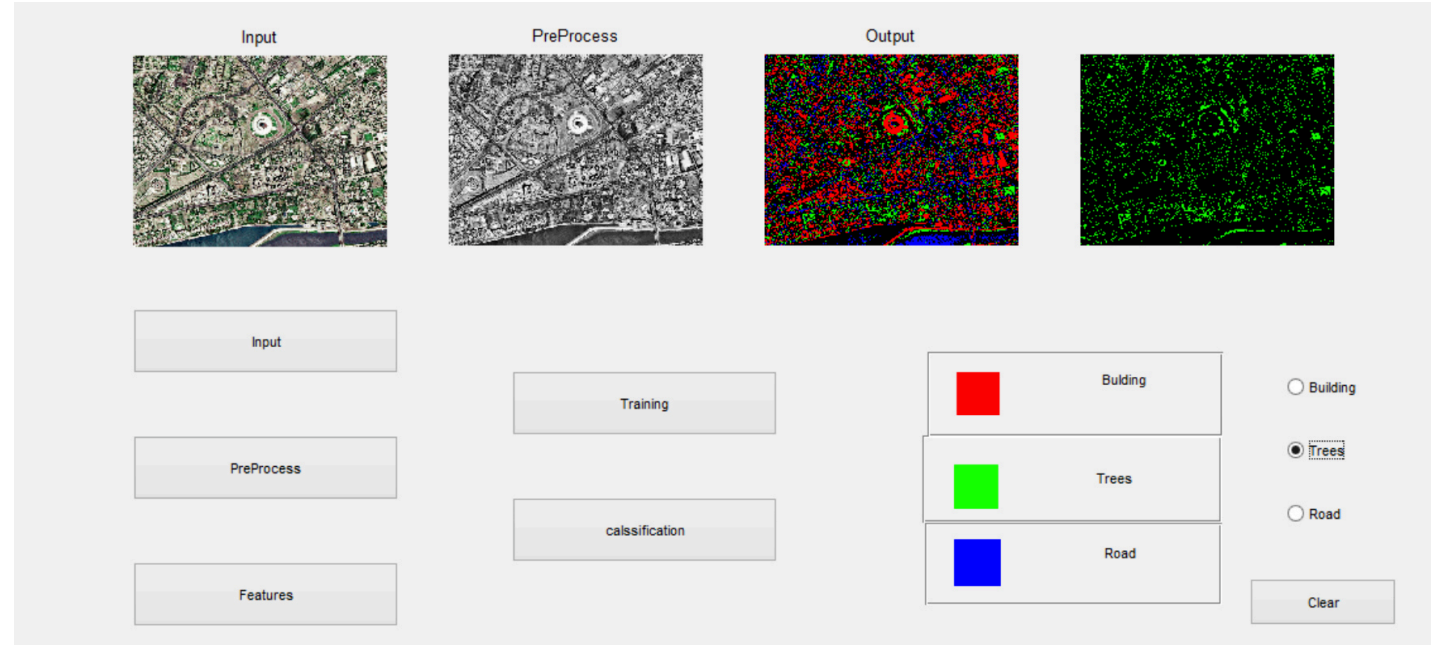

FIG. 4.2. The interest point has been detected with three objects Red (Building), Green (Tree) and Blue (Road)

\section{REFERENCES}

[1] J. M. Bioucas-Dias et Al., "Hyperspectral Remote Sensing Data Analysis And Future Challenges," IEEE Geosci. Remote Sens. Mag., vol. 1, no. 2, pp. 6-36, Jun. 2013.

[2] J. M. Bioucas-Dias et al., "Hyperspectral Unmixing Overview: Geometrical, Statistical, And Sparse Regression-Based Approaches," IEEE J. Sel. Topics Appl. Earth Observ. Remote Sens., vol. 5, no. 2, pp. 354-379, Apr. 2012.

[3] G. G. Wilkinson, "Results And Implications Of A Study Of Fifteen Years Of Satellite Image Classification Experiments," IEEE Trans. Geosci. Remote Sens., vol. 43, no. 3, pp. 433-440, Mar. 2005.

[4] G. M. Foody, "Hard And Soft Classifications By A Neural Network With A Non-Exhaustively Defined Set Of Classes," Int. J. Remote Sens., vol. 23, no. 18, pp. 3853-3864, Jan. 2002.

[5] X. Ceamanos, S. Douté, B. Luo, F. Schmidt, G. Jouannic, and J. Chanussot, "Intercomparison And Validation Of Techniques For Spectral Unmixing Of Hyperspectral Images: A Planetary Case Study," IEEE Trans. Geosci. Remote Sens., vol. 49, no. 11, pp. 4341-4358, Nov. 2011.

[6] R. Heylen, D. Burazerovic, and P. Scheunders, "Fully Constrained Least Squares Spectral Unmixing By Simplex Projection," IEEE Trans. Geosci. Remote Sens., vol. 49, no. 11, pp. 4112-4122, Nov. 2011.

[7] M.D. Iordache, J. Bioucas-Dias, and A. Plaza, "Sparse Unmixing Of Hyperspectral Data," IEEE Trans. Geosci. Remote Sens., vol. 49, no. 6, pp. 2014-2039, Jun. 2011.

[8] J. Tatem, H. G. Lewis, P. M. Atkinson, "Super Resolution Target Identification From Remotely Sensed Images Using A Hopfield Neural Network," IEEE Trans. Geosci. Remote Sens., vol. 39, no. 4, pp. 781-796, Apr. 2001.

[9] P. M. AtKinson, "Mapping sub-pixel boundaries from remotely sensed images," Innovations in GIS IV. London, U.K.: Taylor \& Francis, 1997, ch. 12, pp. 166-180.

[10] D. Song And D. TAO, "Biologically inspired feature manifold for scene classification," IEEE Trans. Image Process., vol. 19, no. 1, pp. 174-184, Jan. 2010.K. Huang, D. Tao, Y. Yuan, "Biologically Inspired Features For Scene Classification In Video Surveillance," IEEE Trans.Syst. Man, Cybern. Part B, Cybern., vol. 41, no. 1, pp. 307-313, Feb. 2011.

[11] M. Muad And G. M. Foody, "Super-resolution mapping of lakes from imagery with a coarse spatial and fine temporal resolution," Int. J. Appl.Earth Observ. Geoinf., vol. 15, pp. 79-91, Apr. 2012.

[12] A. Zia, J. Liang, J. Zhou and Y. GaO, "3D Reconstruction from Hyperspectral Images," 2015 IEEE Winter Conference on Applications of Computer Vision, Waikoloa, HI, 2015, pp. 318-325.

[13] D. Lu And Q. Weng, "A Survey Of Image Classification Methods And Techniques For Improving Classification Performance," International Journal of Remote Sensing, vol. 28, no. 5, pp. 823-870, 2007.

[14] X. Gigandet, M. Cuadra, A. Pointet, L. Cammoun, R. Caloz, and J.-P. Thiran, "Region-Based Satellite Image Classification: Method And Validation," ICIP 2005., vol. 3, pp. III-832-5, September 2005.

[15] J. Lee And T. A. Warner, "Image Classification With A Region Based Approach In High Spatial Resolution Imagery," in International Archives of Photogrammetry, Remote Sensing and Spatial Inf. Sciences, Istanbul, Turkey, July 2004, pp. $181-187$.

[16] S. Pavithra. "Agile Segmentation and Classification for Hyper Spectral Image Using Harris Corner Detector". International Journal of Scientific Research196 International Journal Of Scientific Research Volume : 4, Issue : 7 , ISSN No 2277 8179 ,July 2015

[17] Katartzis, I. Vanhamel, and H. Sahli, "A Hierarchical Markovian Model For Multiscale Region-Based Classification Of Vector-Valued Images," IEEE Transactions on Geoscience and Remote Sensing, vol. 43, no. 3, pp. 548-558, March 2005.

[18] SathiYavani. A S. Pavithra, "Active Segmentation And Classification For Hyper Spectral Image Using Back Propagation" 
ARPN Journal of Engineering and Applied Sciences VOL. 10, NO. 16, September 2015 ISSN 1819-6608.

[19] Suarez, Patricia \& Sappa, Angel \& Vintimilla, Boris. Adaptive Harris Corner Detector Evaluated with Cross-Spectral Images. . (2018) 10.1007/978-3-319-73450-7_69.

Edited by: Swaminathan JN

Received: Oct 29, 2019

Accepted: Jan 28, 2020 